

\section{Sumário}

\section{Dossier Federalismo}

Forma de Estado: Federalismo e RePartição de CompetênCIAS ...................................... 2

Carlos Bastide Horbach

IMUNIDADE RECÍPROCA E FEDERALISMO: DA CONSTRUÇÃO NORTE-AMERICANA À ATUAL POSIÇÃO Do STF

Fernando Santos Arenhart

JUSTIÇA FISCAL, PAZ TRIBUTÁRIA E OBRIGAÇõES REPUBLICANAS: UMA BREVE ANÁLISE DA DINÂMICA JuRisprudencial tributária do Supremo Tribunal Federal ............................................34

Luís Carlos Martins Alves Jr

Federalismo, estado Federalista e a REVALORIZAÇÃo do mUNicípio: UM NOVO CAMINHO PARA O SÉCULO XXI?

Antonio Celso Batista Minhoto

EfEITOS POLÍtTICO-JURÍdicos DA NÃo INSTITUCIONALIZADA PARADIPLOMACIA NO BRASIL ..........66

Gustavo de Souza Abreu

The Management of Public Natural Resource Wealth.

Paul Rose

A (IN)COMPETÊNCIA DO CONAMA PARA EDIÇÃo DE NORMAS SOBRE LICENCIAMENTO AMBIENTAL: ANÁLISE DE SUA JURIDICIDADE ...................................................................................... 118

André Fagundes Lemos

\section{Artigos sobre outros temas}

TEORÍA DE LA PRESIÓN TRIBUTARIA EN BASE A LA IGUALDAD INTERGENERACIONAL: UNA PERSPECTIVA FINANCIERA Y TRIBUTARIA DEL CASO ARGENTINO.

Luciano Carlos Rezzoagli e Bruno Ariel Rezzoagli

CRÉdito TRIBUTÁRIO: GARANTIAS, PRIVILÉGIOS E PREFERÊNCIAS.

Luís Carlos Martins Alves Júnior

Tributário - O parecer PGFN/CRJ 492/2011 e os efeitos da coisa Julgada inconstitucional em face da segurança jurídica no Estado Democrático de Direito* 
A seguranÇa jurídica administrativa na jurisprudência do Supremo Tribunal Federal: UMA ANÁLISE ACERCA DOS FUNDAMENTOS NORMATIVOS E DOS ARGUMENTOS JURÍDICOS NOS JULGAMENTOS DOS MANDADOS DE SEGURANÇA 24.781 E 25.116 195

Ana Paula Sampaio Silva Pereira

Avaliação legislativa no Brasil: apontamentos para uma nova AgENDA de PESQUiSa SoBRE O MODO DE PRODUÇÃO DAS LEIS.....................................................................229

Natasha Schmitt Caccia Salinas

Políticas públicas, DeVERES Fundamentais E CONCRETIZaÇão de DiReitos Julio Pinheiro Faro

Políticas públicas de guerra Às drogas: o ESTAdo de EXCEÇão E A transiÇão do inimigo SCHMitTiano ao homo SaCER de Agamben João Victor Nascimento Martins

NEW INSTITUTIONS FOR THE PROTECTION OF PRIVACY AND PERSONAL DIGNITY IN INTERNET COMMUNICATION - "INFORMATION BROKER", "PRIVATE CYBER COURTS" AND NETWORK OF CONTRACTS

Karl-Heinz Ladeur

RESPONSABILIDADE CIVIL DECORRENTE DE ERRO MÉDICO.

Edilson Enedino das Chagas e Héctor Valverde Santana

A atual geração de energia elétrica SEgundo a lógica de merCado e SuA Ainda CaraCTERIZAÇÃO COMO SERVIÇO PÚBLICO.

Humberto Cunha dos Santos

EMPRESAS, RESPONSABILIDADE SOCIAL E POLÍTICAS DE INFORMAÇÃo OBRIGATÓRIA NO BRASIL.....

Leandro Martins Zanitelli

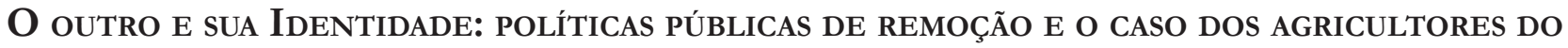
Parque Estadual da Pedra Branca/RJ.

Andreza A. Franco Câmara

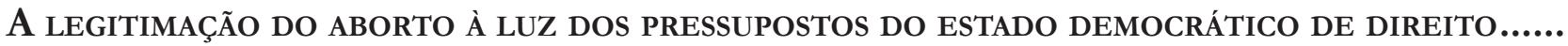

Terezinha Inês Teles Pires

JUSPOSITIVISMO, DISCRICIONARIEDADE E CONTROLE JUDICIAL DE POLÍTICAS PÚBLICAS NO DIREITO BRASILEIRO

Guilherme Valle Brum

A governança transnacional ambiental na Rio +20 . Paulo Márcio Cruz e Zenildo Bodnar 
O QUE É UMA BOA TESE DE DOUTORADO EM DiREITO? UMA ANÁlise A PARTIR DA PRÓPRIA PERCEPÇÃO DOS PROGRAMAS ............................................................................424

Nitish Monebhurrun e Marcelo D. Varella

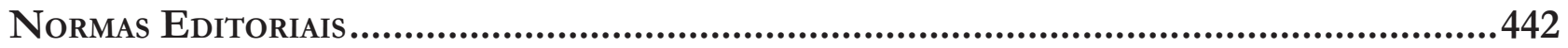

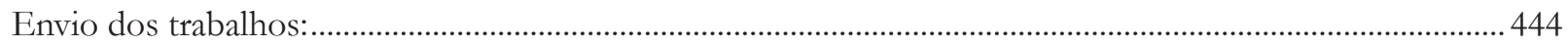




\title{
Empresas, responsabilidade social e políticas de informação obrigatória no Brasil'
}

\section{Corporations, social responsibility and mandated information disclosure policies in Brazil*}

Leandro Martins Zanitelli**

\begin{abstract}
Resumo
O presente artigo examina o potencial e as dificuldades das políticas de informação obrigatória como meio para melhorar o desempenho social e ambiental das empresas no Brasil. Consiste em um trabalho de revisão da literatura acerca das políticas de informação obrigatória e de aplicação do estado da arte da pesquisa às particularidades do capitalismo brasileiro, valendo-se, em relação a esse último ponto, da abordagem conhecida como "variedades de capitalismo" e da tentativa recente de sua aplicação aos países da América Latina. O texto conclui em favor do uso das políticas de informação obrigatória para a promoção da responsabilidade social das empresas, sem deixar, no entanto, de salientar alguns dos obstáculos para o sucesso dessas políticas no caso brasileiro.
\end{abstract}

Palavras-chave: Políticas de informação obrigatória. Responsabilidade social empresarial. Variedades de capitalismo. Brasil.

\section{Abstract}

The article assesses the potential and pitfalls of mandated information disclosure policies as a means to improve the social and environmental performance of corporations in Brazil. It consists of a review of international literature regarding regulation through information and of its application to the Brazilian case, whose particularities are discussed through the lens of the "varieties of capitalism" approach as recently applied to the analysis of Latin American countries' capitalism. The paper finally argues for the use of mandated information regulation as a tool to enhance corporation social responsibility in Brazil, but not without stressing the hazards facing this regulatory strategy in the Brazilian case.

Keywords: Mandated information disclosure. Corporate social responsibility. Varieties of capitalism. Brazil.

1 Agradeço a um revisor anônimo da Revista Brasileira de Políticas Públicas pelos comentários feitos a uma versão anterior deste texto. 


\section{INTRODUÇÃO}

O presente trabalho trata de políticas de informação obrigatória como medidas destinadas a melhorar o desempenho social e ambiental das empresas, com ênfase para a sua aplicação ao caso brasileiro. Como política de informação obrigatória entende-se qualquer medida que, ao invés de impor restrições à atividade empresarial, limite-se a requerer a prestação de informações acerca dessa atividade ou de seu impacto. Um exemplo seria o de uma norma legal que, a fim de combater possível viés racial na admissão de empregados, determine às empresas que divulguem publicamente, a cada ano, o percentual de trabalhadores negros contratados e em exercício de cargo de chefia.

Políticas de informação obrigatória não são, diga-se de saída, uma novidade na regulação da atividade empresarial no Brasil, inclusive no que se refere à regulação que tenha em vista atender a objetivos como o respeito aos direitos humanos e à proteção do meio ambiente. Entre as disposições atualmente em vigor, podem ser citadas as que impõem às empresas o dever de informar aos consumidores sobre as características e, em particular, a periculosidade de produtos, tais como as encontradas no Código de Defesa do Consumidor (Lei 8.078/1990, Arts. 6 $6^{\circ}$ III, $8^{\circ}, 9^{\circ}$ e 31). ${ }^{2}$

Ainda assim, quando comparado ao de outros países, o interesse pela informação obrigatória como meio de regulação da atividade empresarial no Brasil é modesto. ${ }^{3} \mathrm{Na}$ área ambiental, as medidas de informação obrigatória chegam a ser referidas por autores estrangeiros como a "terceira onda" da regulação ambiental, ${ }^{4}$ rivalizando com as políticas tradicionais de "comando-e-controle" e com as soluções ditas "de mercado" (market-based), tais como a tributação e a comercialização do direito de poluir. Um dos exemplos mais conhecidos é o da legislação norte-americana sobre o "Relatório de Despejos Tóxicos" (Toxical Release Inventory, TRI), que obriga indústrias a prestar informações sobre o transporte e a liberação, no meio ambiente, de uma vasta quantidade de substâncias tóxicas. O TRI foi criado na década de 1980 e inspirou medidas similares na União Europeia e em países como Canadá, Austrália, Japão, Coreia do Sul, México e República Tcheca. ${ }^{5}$ No Brasil, em contrapartida, embora a legislação exija a prestação de informações das empresas, sobretudo mediante a entrega de um relatório anual sobre atividades potencialmente poluidoras ou que façam uso de recursos ambientais (Lei 6.938/1981, Art. 17, II; Instrução Normativa/IBAMA 31/2009, Art. 5), as informações obtidas não são divulgadas e, como consequência, acabam impedidas de exercer outra função regulatória que não a de auxiliar as autoridades ambientais na aplicação de medidas de comando-e-controle. ${ }^{6}$

Outra demonstração do desinteresse brasileiro por estratégias de regulação baseadas na informação provém da literatura. Muito embora abundem os estudos sobre deveres de informação atualmente prescritos pela legislação, mormente os do Código de Defesa do Consumidor, são incomuns obras que aventem o uso da informação como solução para novas áreas e examinem, por conseguinte, a conveniência de políti-

2 V. também a Lei 9.294/1996, com determinações sobre informações a serem prestadas por ocasião da venda de produtos oriundos do tabaco, bebidas alcoólicas, medicamentos, terapias e defensivos agrícolas; e o Decreto 4.680/2003, sobre alimentos que contenham OGMs (organismos geneticamente modificados).

3 É importante ressalvar, não obstante, a recente entrada em vigor da Lei de Acesso à Informação (Lei 12.527/11). Embora essa lei tenha por objeto apenas a divulgação de informação, por agentes públicos e organizações privadas sem fins lucrativos, que contém com recursos públicos, ela pode servir indiretamente à divulgação de informações sobre a atividade empresarial ao permitir acesso a informações de órgãos do governo que sejam reguladores das empresas. Além disso, o eventual sucesso da referida lei pode servir de estímulo para o surgimento, no futuro, de medidas de regulação pelas informações endereçadas às empresas.

4 TIETENBERG, Tom. Disclosure strategies for pollution control. Environmental and Resource Economics, v. 11, n. 3-4, p. 587-602, 1998. 5 DASGUPTA, Susmita; WANG, Hua; WHEELER, David. Disclosure strategies for pollution control. In: TIETENBERG, Tom; FOLMER, Henk (Org.). The International Yearbook of Environmental and Resource Economics: A survey of current issues. Cheltenham: Edward Elgar, 2007, p. 93-119. Enquanto as legislações dos países citados se limitam a exigir informações das empresas, em outros, como Indonésia, Filipinas, China, Vietnã e Índia, a política de regulação por intermédio da informação inclui uma classificação pública das empresas de acordo com o seu impacto sobre o meio ambiente.

6 Outra medida sobre informação na área ambiental que obriga a publicar na internet, trimestralmente, lista com os nomes de infratores e as sanções aplicadas (Decreto 6.514/2008, Art. 149) não vem sendo cumprida pela principal agência governamental em âmbito federal, o IBAMA (Instituto Brasileiro do Meio Ambiente e dos Recursos Naturais Renováveis). 
cas públicas baseadas na informação. ${ }^{7} \mathrm{Na}$ literatura estrangeira, em contrapartida, encontram-se trabalhos dedicados não só a avaliar, em geral, as virtudes (e fragilidades) da informação como meio de regulação, ${ }^{8}$ como a defender a sua aplicação para o enfrentamento de problemas pontuais, tais como o da emissão de gases-estufa ${ }^{9}$ e violação a direitos de trabalhadores. ${ }^{10}$

Este trabalho apresenta uma síntese da literatura sobre regulação mediante informação e avalia esta como meio de melhorar o desempenho social e ambiental das empresas no Brasil. Além de ter em vista os debates sobre a divulgação da informação como meio de regulação, o artigo procura considerar as peculiaridades do caso brasileiro, tentando definir, assim, as chances de sucesso de medidas informativas à luz dessas peculiaridades. Para tanto, faz-se uso da economia política comparada e, em especial, da abordagem conhecida como "variedades de capitalismo". ${ }^{11}$ Essa abordagem tem a vantagem de pôr a empresa, alvo das medidas regulatórias a que o presente trabalho se refere, sob o foco da análise. Além disso, embora os trabalhos pioneiros sobre as variedades de capitalismo tenham se limitado a comparar instituições dos países mais industrializados, como EUA e Alemanha, estudos recentes têm tratado de estender a análise para a América Latina ${ }^{12}$ e o Brasil, ${ }^{13}$ oferecendo um promissor ponto de partida para o estudo da regulação empresarial e de técnicas alternativas de regulação, como as que se baseiam na difusão de informações. ${ }^{14}$

$\mathrm{O}$ artigo é organizado como segue. A seção 1 se dedica à definição de políticas de informação obrigatória e a algumas distinções úteis para a análise. A seção 2 revisa as razões apresentadas na literatura para a regulação baseada na informação, o que tanto inclui as razões para procurar alternativas à regulação tradicional de comando-e-controle como as razões, propriamente ditas, em favor do uso da informação com meio de regulação. A seção 3 trata de duas dificuldades enfrentadas pelas políticas de informação obrigatórias, uma que se refere à confiança nas informações divulgadas e outra quanto à importância dessas informações para gestores e trabalhadores das empresas e outros atores capazes de influenciar as suas decisões. A seção 4 tem

7 Para uma exceção, v. FERNANDES, Iêda; BRITO, Brenda; BARRETO, Paulo. Lições para divulgação da lista de infratores ambientais no Brasil. Revista de Direito Ambiental, v. 50, p. 81-101, abr./jun. 2008. Segundo BLACKMAN, Allen. Alternative pollution control policies in developing countries. Review of Environmental Economics and Policy, v. 4, n. 2, p. 234-253, 2010. p. 234. o uso de estratégias de regulação alternativas à regulação tradicional de comando-e-controle na área ambiental é ainda pouco comum nos países em desenvolvimento, embora seja de notar uma tendência à proliferação dessas estratégias, em especial das que se baseiam na disseminação da informação.

8 V., e.g., BEN-SHAHAR, Omri; SCHNEIDER, Carl E. The failure of mandated disclosure. University of Michigan Law School, Empirical Legal Studies Center, Working Paper n. 10-008, 2010. Disponível em: <https://www.law.umich.edu/centersandprograms/ lawandeconomics/abstracts/2010/Documents/10-008benshahar.pdf >. Acesso em: 9 jul. 2012; HESS, David. The three pillars of corporate social reporting as new governance regulation: disclosure, dialogue and development. Michigan Ross School of Business, Working Paper, 2008. Disponível em: <http://deepblue.lib.umich.edu/bitstream/2027.42/60425/1/1112-DHess.pdf>. Acesso em: 10 ago. 2012; VAN ERP, Judith. Reputational sanction in private and public regulation. Erasmus Law Review, v. 1, n. 5, p. 145-162, 2008; WEIL, David; FUNG, Archon; GRAHAM, Mary et al. The effectiveness of regulatory disclosure policies. Journal of Policy Analysis and Management, v. 25, n. 1, p.155-181, 2006; STEPHAN, Mark. Environmental information disclosure programs: they work, but why? Social Science Quarterly, v. 83, n. 1, mar. 2002; KARKKAINEN, Bradley C. Information as environmental regulation: TRI and performance benchmarking, precursor to a new paradigm? Georgetown Law Journal, v. 89, p. 257-370, 2001.

9 SCHATZ, Andrew. Regulating greenhouse gases by mandatory information disclosure. Virginia Environmental Law Journal, v. 26 , p. 335-393, 2008.

10 ESTLUND, Cynthia. Just the facts: the case for workplace transparency. Stanford Law Review, v. 63, p. 351-408, 2011; DOOREY, David J. Who made that? Influencing foreign labour practices through reflexive domestic disclosure regulation. Osgoode Hall Law Journal, v. 43, p. 353-405, 2005.

11 HALL, Peter A.; SOSKICE, David. An introduction to varieties of capitalism. In: HALL, Peter A.; SOSKICE, David (Org.). Varieties of Capitalism: the institutional foundations of comparative advantage. Nova York: Oxford University Press, 2001, p. 1-68.

12 SCHNEIDER, Ben Ross. Comparing capitalisms: liberal, coordinated, network, and hierarchical varieties. 2008. Disponível em: <http://www.ces.fas.harvard.edu/events/papers/Schneider_Comparing_Capitalisms.pdf>. Acesso em: 20 jun. 2012; SCHNEIDER, Ben Ross. Hierarchical market economies and varieties of capitalism in Latin America. Journal of Latin American Studies, v. 41, n. 3, p. 553-575, 2009.

13 NÖLKE, Andreas. A "BRIC" variety of capitalism and social inequality: the case of Brazil. Revista de Estudos e Pesquisas sobre as Américas, v. 4, n. 1, p. 1-14, 2010.

14 Para um ensaio de análise das práticas de responsabilidade social empresarial no Brasil à luz da abordagem das variedades de capitalismo, v. ZANITELLI, Leandro Martins. Capitalismo brasileiro e responsabilidade social empresarial. Sequência, v. 34, n. 66, p. 83-112, 2013. 
em vista certas particularidades do capitalismo brasileiro e suas implicações para as políticas de informação obrigatória, e a seção final resume o trabalho com recomendações acerca do uso das políticas em questão no Brasil.

\section{Políticas de INFORMAÇÃo obRigatóRIA: Definição e distinções}

Políticas de informação obrigatória são as que impõem a certos atores a prestação de informações, diferindo, assim, das políticas tradicionais de comando-e-controle, que se caracterizam por exigir dos atores regulados certa conduta (outra que não a mera prestação de informação) ou resultado. Tome-se, para exemplificar, a conhecida política norte-americana do TRI, que atribui a empresas a obrigação de informar sobre transporte e liberação de substâncias tóxicas. Essa medida claramente se distingue de uma medida de comando-e-controle, já que não trata de proibir ou limitar o uso industrial de substâncias tóxicas, mas apenas de requerer das empresas a informação a respeito desse uso.

Políticas de informação obrigatória e de comando-e-controle não são excludentes. Pode-se, por exemplo, proibir certa atividade nociva ao meio ambiente e, ao mesmo tempo, determinar que empresas informem sobre a eventual ocorrência dessa atividade. É preciso, assim, considerar a conveniência tanto do uso exclusivo da informação com finalidade regulatória como de seu uso combinado com outras medidas.

Uma distinção importante é entre políticas que se limitam a obrigar empresas a prestar informação e políticas que prevejam, além disso, a reunião das informações prestadas de modo a facilitar a comparação do desempenho de diferentes empresas. A legislação brasileira de proteção ao consumidor, por exemplo, impõe amplos deveres de informação com o intuito de ajudar os consumidores em suas tomadas de decisão, mas não reúne as informações prestadas pelos empresários. Embora a tarefa de reunir informações possa ser (e, por vezes, de fato é) executada por outros atores (por exemplo, associações de consumidores, sites de compras), ela não constitui, de um modo geral, parte das políticas públicas brasileiras de informação ao consumidor, já que não fica legalmente incumbida aos órgãos de proteção ao consumidor ou a quaisquer outros agentes. ${ }^{15}$ No caso do TRI norte-americano, em contrapartida, a informação é não apenas apresentada pelas empresas a um órgão governamental, a Agência de Proteção Ambiental (Environmental Protection Agency, EPA), como também se encarrega este órgão de manter um banco com as informações recolhidas, o que facilita a realização de comparações. ${ }^{16}$

Outra distinção é entre políticas de informação obrigatória que exigem a prestação de informação a órgãos governamentais, mas permitem ou imponham manter tal informação sob sigilo, e políticas de informação e divulgação obrigatória da informação. Embora em ambos os casos se possa falar em políticas de informação obrigatória, as políticas do primeiro grupo servem apenas para instruir a ação governamental e não contam, pois, como as do segundo, com outros atores (como sindicatos, associações locais, ONGs em geral) para a realização dos fins a que se propõem.

Por último, observe-se que embora as políticas de informação examinadas neste artigo sejam ditas “obrigatórias", o que sugere se tratar de políticas estatais, boa parte da análise encontrada a seguir é aplicável igualmente à informação espontaneamente prestada (isto é, prestada sem exigência legal) pelas empresas, seja individualmente, seja em decorrência de acordo celebrado com outras empresas ou outros atores (inclusive o próprio Estado).

15 Uma exceção é a informação sobre as taxas de juros e encargos bancários, reunida e divulgada pelo Banco Central do Brasil em seu site. V. <http://www.bcb.gov.br/?LAI>. Acesso em: 16 jul. 2012.

16 KARKKAINEN, Bradley C. Information as environmental regulation: TRI and performance benchmarking, precursor to a new paradigm? p. 286-287. 


\section{JUSTIFICATIVA}

Embora não se refira à informação obrigatória em si mesma, parte da justificativa para estratégias de regulação baseadas na informação tem a ver com os defeitos da regulação de comando-e-controle. Entre os méritos da regulação pela informação estariam, assim, os de não apresentar alguns dos inconvenientes da solução regulatória tradicional.

À regulação de comando-e-controle podem ser atribuídos os seguintes defeitos. ${ }^{17}$ Primeiramente, como estratégia de regulação que prescreve às empresas certo comportamento ou resultado, a regulação de comando-e-controle está sujeita a exigir demais (isto é, a impor mais restrições à atividade empresarial do que o desejável) ou de menos (problema da exatidão). Esse risco é agravado à medida que a tarefa de regular com exatidão se complique, e a complicação aumenta com a amplitude do fim regulatório e a diversidade das situações a regrar. Por exemplo, se não é fácil determinar a quantidade tolerável de emissão de gases-estufa para uma determinada indústria, menos fácil ainda é determinar o nível tolerável dessa e de outras atividades poluidoras não apenas para uma, mas para dezenas de indústrias diferentes. Em particular no caso da regulação da atividade industrial, outro fator a complicar a tarefa de legislar com acuidade é o rápido avanço da técnica, em virtude do qual uma disposição regulatória, mesmo que não o seja inicialmente, pode em pouco tempo se tornar desajustada. ${ }^{18}$

O problema da exatidão pode ser enfrentado mediante a constituição de agências governamentais com poderes e expertise para criar normas adequadas e adaptá-las rapidamente quando for preciso, mas isso põe em evidência outro inconveniente da regulação de comando-e-controle, a saber, o dos custos a arcar com a confecção de normas (problema dos custos com a elaboração de normas). Além disso, e independentemente da sua exatidão, a aplicação das disposições regulatórias de comando-e-controle não se faz sem custos, seja com a fiscalização dos atores regulados, seja com eventuais processos administrativos e judiciais (problema dos custos com a aplicação de normas).

Repare-se agora como a regulação baseada em informação faz frente aos problemas listados. O primeiro problema, da exatidão, é em parte superado no caso da regulação pela informação, já que esse modo de regular não prescreve às empresas qualquer comportamento (afora, claro, o de informar) e, portanto, não corre o risco de exigir das empresas mais do que o que seria desejável. Por outro lado, as políticas públicas de informação obrigatória contam, como se verá adiante, com outros fatores que não a ameaça de sanção legal (por exemplo, as expectativas de consumidores e trabalhadores) para modificar o comportamento das empresas. Assim, o problema da exatidão das políticas de informação obrigatória deve ser examinado à luz de outros fatores de convencimento ou coação que não a lei mesma, fatores esses que, a exemplo dos regramentos de comando-e-controle, podem acabar restringindo a atividade empresarial em uma medida aquém ou além da apropriada.

Ainda no que diz respeito ao problema da exatidão, pode-se afirmar que a grande diferença entre a regulação de comando-e-controle e a regulação baseada na informação decorre do fato de a primeira limitar diretamente a atividade das empresas, enquanto que os limites decorrentes da segunda não são limites impostos pela legislação em si, mas, quando muito, provocados por ela, como no caso de uma empresa que, depois da divulgação de informação negativa sobre mão de obra escrava entre seus fornecedores, toma providências a fim de não ser castigada por investidores ou consumidores éticos. Essa diferença entre limites diretos e

17 Os inconvenientes da regulação de comando-e-controle são esmiuçados em trabalhos nos quais se defendem soluções "de mercado" para problemas ambientais, como incentivos fiscais e a comercialização do direito de poluir. V., e.g., TIETENBERG, Thomas H. Emissions trading: an exercise in reforming pollution policy. Washington, DC: Resources for the Future, 1985; ACKERMAN, Bruce A.; STEWART, Richard B. Reforming environmental law. Stanford Law Review, v. 37, p. 1333-1366, 1985.

18 O problema da exatidão não desaparece quando as normas promulgadas são acentuadamente vagas, já que então se passa a enfrentá-lo por ocasião da aplicação da norma. A vagueza (ainda que quiçá indesejável por outras razões) pode, não obstante, tornar o conteúdo da regulação mais exato à medida que, em comparação com o órgão encarregado de criar as normas, o órgão aplicador esteja mais bem equipado ou consiga responder mais rapidamente a uma alteração das circunstâncias. 
indiretos é também uma diferença entre limites determinados, em última instância, pelo Estado (os limites da regulação de comando-e-controle) e limites oriundos de pressões sociais difusas e até autoimpostos pelas empresas (os da regulação pela informação); estes podem então superar aqueles em precisão, à medida que essa precisão seja uma função da participação de maior número de atores, inclusive dos gestores e trabalhadores, na definição das obrigações sociais da empresa.

Em relação ao segundo problema, dos custos com a elaboração de normas, é plausível afirmar que os custos em questão sejam, em geral, atenuados pela regulação baseada na informação. Embora essa regulação também se componha de normas definidoras do que deve ser informado e por quem (bem como de sanções para o eventual descumprimento), a tendência é que essas normas demandem menos tempo e expertise para a sua elaboração do que as normas de comando-e-controle. Normas segundo as quais as empresas devem informar sobre a contratação de trabalhadores portadores de deficiência, por exemplo, são aparentemente mais simples de criar do que normas que obriguem as empresas a contratar esses trabalhadores, já que as segundas provavelmente precisam considerar particularidades, tais como o tamanho da firma e o setor de sua atividade.

Os custos com a aplicação de normas se traduzem, no caso da regulação pela informação, em custos com a prestação da informação, verificação de sua veracidade e de aplicação de sanções. A magnitude desses custos varia, assim, de acordo com o volume e frequência da informação exigida e com a frequência com a qual a veracidade dessa informação é verificada. Por causa dessa variação, é difícil fazer uma comparação a priori no que se refere aos custos de aplicação, entre as políticas de informação obrigatória e as tradicionais de comando-e-controle. Não obstante, é válido especular que, sendo o custo com o cumprimento das normas de informação para as empresas geralmente inferior ao das normas de comando-e-controle, as violações sejam menos frequentes no primeiro caso, o que enseja uma redução tanto dos custos de fiscalização quanto dos de aplicação de sanções.

Se, por um lado, as políticas de informação obrigatória possuem a vantagem de simplificar a tarefa de elaboração de normas e de não impor (não, ao menos, diretamente) restrições indesejáveis à atividade empresarial, resta, por outro lado, a questão de saber como essas políticas, que nada exigem senão a prestação de informações, são capazes de alterar o comportamento empresarial. Essa é uma questão sobre a qual a literatura estrangeira acerca da regulação pela informação tem frequentemente se debruçado.

Um argumento comum é o de que as políticas de informação obrigatória tornam-se eficazes porque, paradoxalmente, servem para informar os próprios gestores das empresas. ${ }^{19}$ Parte-se da premissa de que algumas informações acerca de uma organização sejam desconhecidas por aqueles que a comandam. Assim, mesmo que a lei não faça outras exigências, o simples fato de impor a obrigação de informar poderia surtir resultado ao levar informações novas sobre a empresa aos seus dirigentes.

Outra hipótese é que a exigência da informação contribua para o "desenvolvimento moral" da companhia. ${ }^{20}$ Ainda que uma política de informação obrigatória não imponha, por si só, alteração no comportamento da empresa, a mera atribuição do dever de informar pode ter o condão de tornar salientes certas normas sociais $^{21}$ e, em consequência, modificar o comportamento de diretores e empregados. Uma medida que

19 KARKKAINEN, Bradley C. Information as environmental regulation: TRI and performance benchmarking, precursor to a new paradigm? p. 295-305; STEPHAN, Mark. Environmental information disclosure programs: they work, but why? p. 194; LEE, E. Information disclosure and environmental regulation: green lights and gray areas. University of Hong Kong, 2010. Disponível em: <http:// hub.hku.hk/bitstream/10722/125310/1/Content.pdf?accept=1>. Acesso em: 9 ago. 2012, p. 8-10; para evidências empíricas, v. LEE, E. Information disclosure and environmental regulation: green lights and gray areas, p. 23-27; BLACKMAN, Allen; AFSAH, Shakeb; RATUNANDA, Damayanti. How do public disclosure pollution control programs work: evidence from Indonesia. Human Ecology Review, v.11, n. 3, p.235-246, 2004; LANOU, Steven M. Production and organization learning: towards a new orientation for environmental policy. Massachusetts Institute of Technology, jun. 1998. Disponível em: <http://web.mit.edu/dusp/etpp/content/ projects/papers/Lanou\%20MIT\%20MCP\%20Thesis\%201998.pdf> . Acesso em: 17 jul. 2012.

20 HESS, David. The three pillars of corporate social reporting as new governance regulation: disclosure, dialogue and development, p. 30-35.

21 CIALDINI, Robert B.; RENO, Raymond R.; KALLGREN, Carl A. A focus theory of normative conduct: recycling the con- 
requeira informações sobre o percentual de trabalhadores portadores de deficiência em uma empresa pode não só revelar o quão raramente esses trabalhadores são contratados como também tornar saliente, entre administradores e empregados, uma norma social avessa à discriminação e, em consequência, provocar um comportamento conforme a essa norma, aumentando a frequência com que trabalhadores com deficiência são admitidos.

Outros argumentos ressaltam o impacto que a informação prestada pode ter sobre outros atores que não os dirigentes da companhia e a importância de pressões externas para uma mudança na conduta empresarial. A ideia é bem representada pela expressão "ciclo de ação" (action cycle) usada em estudo de Weil e colegas. ${ }^{22}$ Informados sobre a atividade de uma corporação, outros atores, como consumidores e investidores, ajustariam seu comportamento à informação recebida e, ao fazê-lo, induziriam, reflexamente, uma alteração no comportamento dos gestores da companhia. ${ }^{23}$ A reação de outros atores às informações sobre o desempenho social da empresa tanto pode ser motivada pelo autointeresse como por preferências sociais (por exemplo, no caso de consumidores éticos ou "verdes"). Em outras palavras, uma política de informação obrigatória pode ter impacto sobre o comportamento desses atores por muni-los de informações relevantes para escolhas autointeressadas ou devido a preferências não egoístas de que a divulgação da informação contribui para satisfazer ou até criar (considerando-se, uma vez mais aqui, o efeito de saliência que a exigência da informação é capaz de suscitar).

Assim, à medida que o sucesso de políticas de informação obrigatória dependa do impacto da informação sobre outros atores que não os gestores da companhia, importa verificar quem são esses atores e por que a informação pode influir em suas decisões. Considere-se, em primeiro lugar, o caso dos investidores. A divulgação de informações sobre o desempenho social e ambiental de uma corporação pode importar para essas pessoas devido a uma preferência por empresas socialmente responsáveis. ${ }^{24}$ Outra hipótese é que mesmo investidores interessados apenas em maximizar o valor de sua carteira sejam atraídos por empresas com bom desempenho social e ambiental, seja por temerem a reação de outros atores, como consumidores e autoridades governamentais, à divulgação de informações negativas sobre uma empresa, seja por tratarem a informação negativa como sinal de risco ou gestão inábil, com consequências indesejáveis em outras áreas além daquela a qual a informação se refere. ${ }^{25}$

Há inúmeros trabalhos empíricos investigando a relação entre a informação quanto ao desempenho social e ambiental das corporações e às decisões de investidores. ${ }^{26}$ A evidência oriunda desses trabalhos é mista, o que, se por um lado sugere a possibilidade de a informação modificar o comportamento dos investidores, por outro mostra a necessidade de perquirir sobre as condições para que isso se verifique.

cept of norms to reduce littering in public places. Journal of Personality and Social Psychology, v. 58, n. 6, p. 1015-1026, 1990.

22 WEIL, David; FUNG, Archon; GRAHAM, Mary et al. The effectiveness of regulatory disclosure policies.

23 A rigor, uma alteração nos rumos da empresa pode não depender de que outros atores reajam, de fato, à informação divulgada, mas apenas de que os dirigentes da companhia prevejam essa reação e tratem, assim, de evitá-la.

24 MACKEY, Alison; MACKEY, Tyson B.; BARNEY, Jay B. Corporate social responsibility and firm performance: investor preferences and corporate strategies. Academy of Management Review, v. 32, n. 3, p.817-835, 2007.

25 KONAR, Shameek; COHEN, Mark A. Information as regulation: the effect of community right to know laws on toxic emissions. Journal of Environmental Economics and Management, v.32, p. 109-124, 1997. p. 112-113; KARKKAINEN, Bradley C. Information as environmental regulation: TRI and performance benchmarking, precursor to a new paradigm?, p. 324-325; STEPHAN, Mark. Environmental information disclosure programs: they work, but why?, p. 194.

26 LEE, E. Information disclosure and environmental regulation: green lights and gray areas; GUPTA; Shreekant; GOLDAR, Bishwanath. Do stock markets penalize environment-unfriendly behaviour? Evidence from India. Ecological Economics, v. 52, p. 81-95, 2005; DASGUPTA, Susmita; LAPLANTE, Benoit; MAMINGI, Nlandu. Pollution and capital markets in developing countries. Journal of Environmental Economics and Management, v. 42, p. 310-335, 2001; KONAR, Shammek; COHEN, Mark A. Does the market value environmental performance? The Review of Economics and Statistics, v. 83, n. 2, p. 281-289, 2001; HAMILTON, James T. Pollution as news: media and stock market reactions to the Toxic Releases Inventory data. Journal of Environmental Economics and Management, v. 28, p. 98-113, 1995; LANOIE, Paul; LAPLANTE, Benoit; ROY, Maité. Can capital markets create incentives for pollution control? Ecological Economics, v. 26, p. 31-41, 1998; SHANE, Philip B.; SPICER, Barry H. Market response to environmental information produced outside the firm. The Accounting Review, v. 98, n. 3, p. 521-538, jul. 1983. 
Outro ator cujo comportamento pode ser influenciado pela informação (e que é capaz, por sua vez, de influenciar as decisões dos gestores das empresas) é o consumidor. A exemplo do que acontece com os investidores, a informação pode ser importante tanto para consumidores autointeressados como para aqueles com preferências altruístas. Um exemplo de informação relevante para consumidores do primeiro grupo é a informação sobre os riscos de um produto, enquanto consumidores do segundo grupo podem ser levados a decidir de acordo com o desempenho social ou ambiental de uma corporação. Sobre essa última hipótese, no entanto, há robustas evidências de um desacordo entre "atitude" e comportamento dos consumidores. ${ }^{27}$ Aparentemente, enquanto são muitos os consumidores que estão dispostos a decidir pela compra de produtos verdes ou éticos, é bem menor o número dos que de fato o fazem.

Hipóteses análogas podem ser levantadas em relação à influência da informação sobre trabalhadores. Para um trabalhador, a informação sobre a responsabilidade social de uma empresa (ou a falta dela) pode ser vital ainda quando a decisão sobre o trabalho seja uma decisão exclusivamente autointeressada. ${ }^{28}$ Por exemplo, a notícia sobre violações a direitos humanos, ocorridas no local de trabalho, é relevante para atuais e potenciais trabalhadores, devido à informação que transmite acerca dos riscos do emprego. ${ }^{29}$ Não é de descartar, além disso, que trabalhadores simplesmente prefiram empresas com comportamento socialmente responsável, e que sua decisão de se empregar, portanto, seja influenciada pela informação sobre o desempenho social de uma empresa, independentemente das consequências desse desempenho para os trabalhadores mesmos. ${ }^{30}$

Uma empresa ainda pode reagir à divulgação de informações negativas sobre o seu desempenho social com o intuito de evitar que essas informações provoquem uma intervenção governamental mais incisiva, já que as políticas de informação obrigatória podem ser percebidas pelas empresas como medidas destinadas a chamar à atenção para as necessidades de regulação mais prementes e a dar lugar, caso elas permaneçam inertes, a medidas mais agressivas. ${ }^{31}$ Esse temor da intervenção governamental pode ganhar força nos casos em que a informação difundida seja usada por outros atores (por exemplo, ONGs e órgãos de imprensa) para exigir providências das autoridades.

No que se refere, em particular, à divulgação de informações sobre o impacto ambiental das empresas, cogita-se ainda de um efeito da informação sobre o comportamento dos habitantes de localidades sujeitas aos riscos ou danos ambientais decorrente da atividade empresarial. A ideia é que, munidas de informação, as vítimas da poluição seriam motivadas a superar eventuais problemas de ação coletiva e a atuar para obter providências das próprias firmas ou das autoridades. ${ }^{32}$

27 CARRINGTON, Michal J.; NEVILLE, Benjamin A.; WHITWELL, Gregory J. Why ethical consumers don't walk their talk: Towards a framework for understanding the gap between the ethical purchase intentions and actual buying behaviour of ethically minded consumers. Journal of Business Ethics, v. 97, p. 139-158, 2010, e referências lá encontradas; para evidências no Brasil, v. BARCELLOS, Marcia Dutra de; KRYSTALLIS, Athanasios; SAAB, Maria Estela de Melo et al. Investigating the gap between citizens' sustainability attitudes and food purchasing behaviour: empirical evidence from Brazilian pork consumers. International Journal of Consumer Studies, v.35, p. 391-402, 2011.

28 KARKKAINEN, Bradley C. Information as environmental regulation: TRI and performance benchmarking, precursor to a new paradigm? p. 325; ESTLUND, Cynthia. Just the facts: the case for workplace transparency, p. 369-373.

29 Como argumenta KARKKAINEN, Bradley C. Information as environmental regulation: TRI and performance benchmarking, precursor to a new paradigm?, p. 325, a informação sobre riscos ambientais pode influenciar as decisões de trabalhadores que prezem pelo próprio bem-estar e pelo de seus familiares no caso em que a decisão sobre o emprego os obrigue a viver em área sob risco.

30 BACKHAUS, Kristin B.; STONE, Brett A.; HEINER, Karl. Exploring the relationship between corporate social performance and employer attractiveness. Business \& Society, v. 41, n. 3, p. 292-318, set. 2002; GREENING; Daniel W.; TURBAN, Daniel B. Corporate social performance as a competitive advantage in attracting a quality workforce. Business \& Society, v. 39, n. 3, p. 254-280, set. 2000; SCHATZ, Andrew. Regulating greenhouse gases by mandatory information disclosure, p. 376.

31 KARKKAINEN, Bradley C. Information as environmental regulation: TRI and performance benchmarking, precursor to a new paradigm? p. 310-312.

32 KARKKAINEN, Bradley C. Information as environmental regulation: TRI and performance benchmarking, precursor to a new paradigm? p. 316-323; LEE, E. Information disclosure and environmental regulation: green lights and gray areas, p. 7-8. 


\section{LIMITES}

Se, por um lado, as hipóteses acerca da influência da informação sobre o comportamento empresarial permitem inferir razões para o sucesso de políticas de informação obrigatória, por outro lado elas também sugerem certos limites aos quais essas políticas se sujeitam. Esses limites, examinados mais detalhadamente a seguir, dizem respeito à confiança nas informações prestadas e à importância dessas informações para as decisões dos gestores da companhia e de outros atores capazes de influenciar o comportamento dos primeiros.

A fim de que a informação tenha impacto sobre atores, tais como investidores, consumidores e trabalhadores, é crucial que haja confiança nos dados divulgados. Não se pode esperar, por exemplo, que um consumidor tome a decisão de compra com base no desempenho ambiental de empresas concorrentes se não confia na informação sobre esse desempenho. Note-se, quanto a isso, que uma informação na qual se confia não é, necessariamente, uma informação verdadeira, bem como, por outro lado, que mesmo uma informação verdadeira pode, por alguma razão, deixar de despertar confiança. No caso das políticas de informação obrigatória, o fato de a informação ser difundida pela própria empresa a respeito da qual essa informação se refere, e o de a veracidade da informação não ser verificada por terceiros, são possíveis causas para a aludida falta de confiança. ${ }^{33}$ A esse respeito, vale observar que embora um meio de combater a desconfiança acerca das informações difundidas seja o controle da sua veracidade por terceiros (por exemplo, agentes públicos e ONGs), o exercício do controle não apenas eleva o custo de aplicação da medida legal como ainda tem o seu sucesso condicionado à crença do público em sua eficácia.

Em relação à importância da informação, aventou-se anteriormente que a informação resultante de uma política de informação obrigatória é capaz, por si só, de levar a uma alteração do comportamento empresarial. Essa alteração tanto pode ser entendida como alteração estratégica quanto institucional. ${ }^{34}$ Em quaisquer das hipóteses, um impacto sobre a atividade da empresa somente pode ser esperado se a informação for importante para os gestores, variando as razões dessa importância de acordo com o modo de atuação, estratégico ou institucional, que venha a ser verificado. No primeiro caso (alteração estratégica), postula-se que as decisões dos gestores estejam destinadas a alcançar certos objetivos, de maneira que a informação resultante da exigência legal importará à medida que esteja relacionada à realização desses objetivos. Por exemplo, a informação sobre a atividade poluidora de uma empresa pode chamar a atenção dos gestores para a ineficiência do processo de produção, levando-os a agir para aperfeiçoar esse processo e, em consequência, elevar os lucros. ${ }^{35}$ No segundo caso (alteração institucional), tem-se em vista o fato de os gestores estarem sujeitos à influência de normas sociais e a possibilidade de a informação resultante de uma política de informação obrigatória ressaltar a necessidade de conformar o comportamento empresarial a essas normas, independentemente do benefício que isso venha propiciar. Por exemplo, se o cumprimento de um dever legal de informar sobre a proporção de negros e brancos em cargos de chefia em uma organização revelar esmagadora predominância desses últimos, a consequência pode ser a tomada de medidas para combater o viés na ocupação de cargos e ajustar a conduta empresarial à norma social respectiva, isso sem ter, necessariamente, em vista resultado algum (como, por exemplo, o aumento das vendas entre consumidores negros).

A importância da informação para outros atores é também uma condição para o sucesso de políticas de informação obrigatória. ${ }^{36}$ Como observado acima, a informação pode importar tanto por razões de autointeresse, como no caso de potenciais investidores para quem a informação sobre o desempenho ambiental de uma empresa sinalize falhas de gestão, quanto devido a preferências por empresas socialmente responsáveis. Deve-se atentar, ainda, para a possibilidade de certas informações interessarem a alguns grupos de atores, mas não a outros.

33 Para evidências sobre a falsidade de informações prestadas por empresas, v. DE MARCHI, Scott; HAMILTON, James T. Assessing the accuracy of self-reported data: an evaluation of the toxics release inventory. Journal of Risk and Uncertainty, v. 32, p. 57-76, 2006.

34 SUCHMAN, Mark C. Managing legitimacy: strategic and institutional approaches. Academy of Management Review, v. 20, n. 3, p. 571-610, 1995.

35 SCHATZ Andrew. Regulating greenhouse gases by mandatory information disclosure, p. 373.

36 WEIL, David; FUNG, Archon; GRAHAM, Mary et al. The effectiveness of regulatory disclosure policies, p. 161. 
A importância da informação para gestores e outros atores deve ser considerada não só abstratamente, como no contexto dos processos de tomada de decisão. Mesmo que uma informação seja, em si mesma, importante, ela pode se mostrar inócua para a tomada de decisão devido à racionalidade limitada dos atores, ${ }^{37}$ isto é, a limitações de tempo e capacidade cognitiva. ${ }^{38}$ Daí a recomendação de que a informação requerida por políticas de informação obrigatória seja simples, de modo a facilitar o seu uso em processos decisórios. ${ }^{39}$ Assim, além de compreensível para o público a que se destina, defende-se que a informação permita uma comparação entre o desempenho de diferentes empresas, como no caso em que os dados sobre a atividade empresarial dão origem a rankings ou classificações. ${ }^{40}$ Considera-se importante, ainda, que a informação seja prestada e ressaltada no local e hora em que a decisão é tomada. ${ }^{41}$

Uma dificuldade a considerar é o risco de, devido à simplificação, a informação não despertar a confiança do público. Isso pode ocorrer quando, em benefício da simplificação, os dados divulgados acabam sendo indicadores imperfeitos do comportamento da empresa. Exemplar, a esse respeito, é o TRI norte-americano, o qual, apesar do reconhecido sucesso, é tido como um indicador falho do desempenho ambiental das empresas, entre outras razões, porque relata o volume de substâncias tóxicas liberadas, mas nada diz sobre a sua toxicidade e sobre o grau de exposição da população local a essas substâncias. ${ }^{42}$ Outro exemplo é o dos rankings de empresas, que podem transmitir uma informação de fácil manuseio sobre a responsabilidade social das empresas classificadas, mas suscitar desconfiança exatamente devido à ignorância sobre a base de dados da qual esse ranking é oriundo, ou simplesmente sofrer com a falta de confiança do público na organização, pública ou privada, encarregada da sua realização. ${ }^{43}$

\section{0 CASO BRASILEIRO}

A avaliação acerca das chances de sucesso de uma estratégia de regulação como a das políticas de informação obrigatória deve levar em conta as particularidades do local no qual a estratégia será empregada. No que se refere, assim, à regulação empresarial, é útil ter em conta a abordagem conhecida como "variedades de capitalismo", " que se propõe trazer, à luz da discursão, diferenças entre países capitalistas determinadas por características institucionais e pelo impacto das instituições sobre o comportamento das empresas. Entretanto, a atenção às empresas apenas não é suficiente, já que, como visto nas seções anteriores, o sucesso das medidas de informação obrigatória depende, em larga medida, da reação de outros atores, como investidores e consumidores, às informações sobre o desempenho das corporações. Assim, uma avaliação do potencial da regulação baseada na informação também precisa levar em conta particularidades locais no que se refere ao comportamento desses outros atores.

37 SIMON, Herbert A. A behavioral model of rational choice. Quarterly Journal of Economics, v. 69, p. 99-118, fev. 1955.

38 SAGE, William M. Regulating through information: disclosure laws and American health care. Columbia Law Review, v. 99 , p. 1701-1829, 1999. p. 1.728-1.731; BEN-SHAHAR, Omri; SCHNEIDER, Carl E. The failure of mandated disclosure, p. 40-54.

39 SAGE, William M. Regulating through information: disclosure laws and American health care, p. 1736-1743; WEIL, David; FUNG, Archon; GRAHAM, Mary et al. The effectiveness of regulatory disclosure policies, p. 161-162.

40 WEIL, David; FUNG, Archon; GRAHAM, Mary et al. The effectiveness of regulatory disclosure policies, p. 161. Um exemplo é o programa de controle de poluição indonésio, o PROPER (Pollution Control Evaluation and Rating), que divide as empresas em cinco grupos segundo o desempenho ambiental. A cada um dos grupos é atribuída uma cor pela qual as empresas pertencentes ao grupo podem ser reconhecidas pelo público. V. AFSAH, Shakeb; VINCENT, Jeffrey R. Putting pressure on polluters: Indonesia's PROPER Program. Harvard Institute for International Development, 1997. Disponível em: <http://web.worldbank.org/archive/website01004/WEB/IMAGES/PUTTINGP. PDF>. Acesso em: 16 ago. 2012. Há, na China, programa similar. V. WANG, Hua; BI, Jun; WHEELER, David et al. Environmental performance rating and disclosure: China's GreenWatch Program. Journal of Environmental Management, v. 71, p. 123-133, 2004.

41 WEIL, David; FUNG, Archon; GRAHAM, Mary et al. The effectiveness of regulatory disclosure policies, p. 161.

42 KARKKAINEN, Bradley C. Information as environmental regulation: TRI and performance benchmarking, precursor to a new paradigm? p. 332.

43 LEE, E. Information disclosure and environmental regulation: green lights and gray areas, p. 33.

44 HALL, Peter A.; SOSKICE, David. An introduction to varieties of capitalism. 
$\mathrm{Na}$ análise das variedades de capitalismo, existe ênfase nas diferenças entre as chamadas economias de mercado liberais, ou LMEs (liberal market economies), como as dos EUA e Reino Unido, e as economias de mercado com coordenação, ou CMEs (coordinated market economies), como as da Alemanha e Japão. ${ }^{45}$ Enquanto as primeiras se caracterizam pelo predomínio de relações estritamente de mercado, baseadas em preços e tendencialmente mais instáveis, nas economias coordenadas, em contrapartida, as estratégias empresariais se encontram mais frequentemente alicerçadas em "contratos relacionais" ou alianças mais duradouras das empresas entre si e com trabalhadores e investidores.

A literatura recente tem se dedicado a descrever outras variedades de capitalismo além das de LMEs e CMEs, estendendo a abordagem às economias de outros países, inclusive da América Latina. ${ }^{46}$ Nos próximos parágrafos, considerar-se-á a caracterização feita por Schneider ${ }^{47}$ dos países da América Latina como economias de mercado hierárquicas, HMEs (bierarquical market economies) e suas implicações acerca do uso de políticas de informação obrigatória para a melhora do desempenho social e ambiental das empresas no Brasil.

De acordo com Schneider, ${ }^{48}$ uma análise das estratégias empresariais em países da América Latina precisa ter em vista o fato de que parte considerável da atividade empresarial nesses países é exercida por multinacionais estrangeiras e pelos chamados "grupos econômicos", grupos de empresas com atividade diversificada, geralmente submetida ao controle familiar. A proliferação das multinacionais e dos grupos atribui ao capitalismo latino-americano as características que se descrevem a seguir, todas elas relevantes para uma avaliação do potencial de políticas de informação obrigatória.

Uma das mencionadas características é a insignificância das associações empresariais. Na América Latina, o fortalecimento dessas associações é por um lado dificultado pela falta de poder decisório dos gestores locais das empresas multinacionais e, por outro lado, pela diversificação da atuação dos grupos econômicos, que acabam se dedicando a setores de atividades dos quais seus controladores se encontram distantes. Acrescentem-se, ainda, como empecilho a um estreitamento das relações do empresariado local com os representantes das multinacionais, possíveis diferenças de idioma e cultura. ${ }^{49}$

A fragilidade deve ser considerada quando se trata de uma estratégia de regulação que, ao invés da aplicação de sanções, pretende se valer do efeito moralizante e reputacional da informação, já que esse efeito é dependente das relações da empresa com outros atores. É verdade, por um lado, que as alianças entre empresas podem elevar a resistência a qualquer tentativa regulatória, inclusive a políticas de informação obrigatória. Por outro, no entanto, as associações empresariais podem servir como fórum de aprendizado sobre o valor (e a conveniência) da responsabilidade social ${ }^{50}$ e de interlocução das empresas com diferentes stakeholders (ONGs, trabalhadores, consumidores etc.), contribuindo para que a difusão da informação surta o resultado esperado. Para que exerçam esse papel, porém, é importante que as associações consistam em mais do que uma união formal de um grupo de empresários, estando alicerçadas em relações de confiança ou dependência capazes de ensejar o aprendizado mútuo e de fazer frente aos problemas de ação coletiva que frequentemente atravancam a melhora no desempenho social das empresas.

Outra característica das economias de mercado hierárquicas ligada à atuação das multinacionais e dos grupos econômicos diz respeito à governança corporativa. Muitas multinacionais não abrem o capital de suas subsidiárias ao mercado de ações, e os grupos econômicos, quando o fazem, costumam manter o controle nas mãos da

45 HALL, Peter A.; SOSKICE, David. An introduction to varieties of capitalism.

46 SCHNEIDER, Ben Ross. Hierarchical market economies and varieties of capitalism in Latin America; NÖLKE, Andreas. $A$ "BRIC" variety of capitalism and social inequality: the case of Brazil.

47 SCHNEIDER, Ben Ross. Comparing capitalisms: liberal, coordinated, network, and hierarchical varieties; SCHNEIDER, Ben Ross. Hierarchical market economies and varieties of capitalism in Latin America.

48 SCHNEIDER, Ben Ross. Hierarchical market economies and varieties of capitalism in Latin America, p. 6-10.

49 SCHNEIDER, Ben Ross. Hierarchical market economies and varieties of capitalism in Latin America, p. 15.

50 CAMPBELL, John L. Why would corporations behave in socially responsible ways? An institutional theory of corporate social responsibility. Academy of Management Review, v. 32, n. 3, p. 946-967, 2007. p. 959. 
família, o que desestimula a participação de acionistas minoritários. ${ }^{51}$ A consequência é a hierarquização da governança, isto é, a reduzida influência de investidores outros que não os sócios majoritários. Essa é uma característica relevante para as políticas de informação obrigatória porque, como visto anteriormente, parte da pressão externa que a divulgação da informação pode suscitar é justamente aquela que provém dos investidores.

Além dos modos de atuação empresarial e de suas implicações para as relações das empresas entre si e entre investidores, é necessário avaliar as chances de sucesso da regulação pela informação tendo em vista particularidades locais atinentes a atores cujas expectativas são capazes de influenciar o comportamento empresarial, como consumidores e trabalhadores. Referindo-se ao uso de políticas de informação obrigatória para a melhora do desempenho ambiental de empresas em países em desenvolvimento, Blackman ${ }^{52}$ ressalva certas dificuldades enfrentadas nesses países. Tais dificuldades são, além da já aludida insignificância dos mercados de capitais, a tímida demanda dos consumidores por produtos verdes, a falta ou debilidade das organizações não governamentais e a impotência do Estado, a qual levaria as companhias a não temer medidas de intervenção mais duras apesar da divulgação de informações negativas sobre o seu desempenho. ${ }^{53}$

No Brasil e em outros países com tradição de intervencionismo estatal e de políticas de bem-estar social, pode ser também que o papel protagonista do Estado contribua para uma cultura de irresponsabilidade social das empresas. ${ }^{54} \mathrm{~A}$ ideia, em outras palavras, é que, ao se incumbir de tarefas de promoção do bem-estar diretamente (por exemplo, pela educação pública e gratuita e a prestação de assistência à saúde) e indiretamente (pela regulação da atividade empresarial), o Estado acabe por atrair não apenas demandas sobre a sua atividade mesma como também as que se referem à responsabilidade social das empresas. Essas últimas seriam então percebidas como agentes cuja ganância só pode ser freada pela mão forte do Estado e que, no limite, estão até legitimados a atuar exclusivamente em seu próprio benefício.

Embora uma cultura de irresponsabilidade social empresarial não constitua necessariamente um óbice intransponível às políticas de informação obrigatória, é importante ter em mente que o impacto dessas políticas depende da sensibilidade do público às informações sobre o comportamento das empresas. Podese, entretanto, ponderar, a esse respeito, que a indiferença ao desempenho social e ambiental das empresas tanto pode ser causa de insucesso de medidas de regulação pela informação quanto resultado da falta dessas medidas. É possível, em outras palavras, que a divulgação mais frequente de informações acabe fomentando expectativas sobre a atividade das empresas e tenha, assim, impacto sobre essa atividade mesmo em países nos quais uma cultura pública de responsabilidade empresarial seja pouco desenvolvida.

\section{Considerações finais}

Esta última seção reúne em tópicos as principais conclusões do trabalho, apresentando-as como recomendações sobre o uso de políticas de informação obrigatória para a melhora do desempenho social e ambiental das empresas no Brasil.

1) Quando se trata de exigir a prestação de informações pelas empresas, há uma vantagem em exigir informações que ainda não sejam conhecidas pelos gestores das companhias. A informação que é nova para

51 SCHNEIDER, Ben Ross. Hierarchical market economies and varieties of capitalism in Latin America, p. 14; NÖLKE, Andreas. A "BRIC" variety of capitalism and social inequality: the case of Brazil, p. 7-8.

52 BLACKMAN, Allen. Alternative pollution control policies in developing countries, p. 234-235.

53 Um empecilho ao sucesso de políticas de informação obrigatória é a incapacidade dos consumidores para compreender a informação divulgada. Em países cuja escolaridade média é baixa, como no Brasil, esse problema é particularmente grave.

54 Para evidências em favor de uma hipótese similar, segundo a qual práticas de responsabilidade social e empresarial (entendida como atuação espontânea, não legalmente prescrita, das empresas) são mais difundidas em países nos quais a intervenção do Estado, nos mercados, é mais tímida, v. KINDERMANN, Daniel. Why do some countries get CST sooner, and in greater quantity, than others? The political economy of corporate responsibility and the rise of market liberalism across the OECD: 1977-2007. WZB Discussion Paper. Disponível em: <http://ideas.repec.org/p/zbw/wzbkpw/spiii2009301.html>. Acesso em: 8 jun. 2012. 
o gestor pode ter um efeito de aprendizado capaz de levar a uma mudança de comportamento independentemente de pressões externas.

2) Mudanças no comportamento empresarial são facilitadas quando as relações entre empresas, eventualmente mediadas por associações comerciais, são estreitas, já que essas relações submetem os gestores à pressão dos seus pares e contribuem para o aprendizado mútuo acerca do valor (e da conveniência) da responsabilidade social. Dada a costumeira fragilidade das associações empresariais brasileiras, deve-se ter em vista o particular potencial das políticas de informação obrigatória nos casos excepcionais (como, por exemplo, os de algumas empresas ligadas a cooperativas) em que a proximidade entre os empresários é maior. Infelizmente, as uniões entre empresas também podem ser um obstáculo para que as referidas políticas (assim como qualquer outra medida de regulação) sejam instituídas.

3) Considerando-se que a confiança nas informações tornadas públicas é vital para que essas informações influenciem as decisões de atores como investidores, trabalhadores, consumidores e autoridades governamentais, é preferível exigir informações cuja veracidade seja fácil de verificar. Além disso, à falta de recursos públicos disponíveis para que tal verificação ocorra, é importante ter em vista que o sucesso da regulação pela informação pode depender de atores privados, como ONGs e movimentos sociais, capazes de se desincumbir da tarefa. Assim, as políticas de informação obrigatória são mais promissoras nos países e nas áreas que já contenham ou que estejam mais propensas a contar com esses atores.

4) É preferível que a informação seja divulgada de maneira a facilitar a compreensão e a comparação do desempenho de diferentes empresas. Há de se ter cuidado, no entanto, para que a simplicidade não ponha em risco a credibilidade e a relevância dos dados revelados para os seus destinatários, tal como pode ocorrer nos casos em que a informação seja usada para classificar as empresas, mas em que os critérios da classificação não fiquem claros ou sejam intrincados a ponto de enfraquecer a percepção sobre a relação entre a posição de uma empresa no ranking divulgado e o desempenho dessa mesma empresa em áreas específicas de interesse do público.

5) A informação é tão mais apta a ter impacto quanto mais saliente se faça, preferencialmente por ocasião da tomada de decisão. Assim, mesmo que informações, comparando o desempenho social e ambiental de empresas, sejam bem divulgadas pela mídia e estejam permanentemente à disposição do público (por exemplo, na internet), é importante cuidar para que consumidores, investidores e outros atores cujas decisões são relevantes para as empresas tenham a sua atenção atraída para a informação na hora de decidir.

6) Quando se trata de contar com a pressão de atores externos para uma mudança no comportamento empresarial, é indispensável procurar obter das empresas informações que sejam relevantes para esses atores. Para diferentes grupos de atores, as informações que importam podem ser distintas, de modo que a definição de uma política de informação obrigatória também precisa ter em vista o público-alvo da informação, isto é, aquele - ou aqueles grupos de atores - cujas reações às empresas sejam mais sensíveis. No caso de países com governança corporativa hierarquizada, como o Brasil, pode ser recomendável difundir informações que interessem a outros grupos de atores que não investidores, como consumidores e trabalhadores.

7) É crucial para o sucesso de políticas de informação obrigatória destinadas a obter mais responsabilidade social empresarial que haja um ambiente normativo favorável a essa responsabilidade, de modo que a divulgação de informações negativas sobre o desempenho social ou ambiental de uma empresa tenha não apenas o efeito de embaraço sobre gestores e empregados, como o de alavancar pressões (dos próprios empregados, de consumidores, investidores etc.) por mudanças de comportamento. Apesar disso, mesmo nos países em que a expectativa geral quanto à responsabilidade social empresarial seja modesta, as políticas de informação obrigatória não devem ser, ao menos de saída, descartadas, já que podem contribuir para uma mudança na cultura pública. 


\section{REFERÊNCIAS}

ACKERMAN, Bruce A.; STEWART, Richard B. Reforming environmental law. Stanford Law Review, v. 37 , p. 1333-1366, 1985.

AFSAH, Shakeb; VINCENT, Jeffrey R. Putting pressure on polluters: Indonesia's PROPER Program. Harvard Institute for International Development, 1997. Disponível em: <http://web.worldbank.org/archive/website01004/WEB/IMAGES/PUTTINGP.PDF>. Acesso em: 16 ago. 2012.

BACKHAUS, Kristin B.; STONE, Brett A.; HEINER, Karl. Exploring the relationship between corporate social performance and employer attractiveness. Business \& Society, v. 41, n. 3, p. 292-318, set. 2002.

BARCELLOS, Marcia Dutra de et al. Investigating the gap between citizens' sustainability attitudes and food purchasing behaviour: empirical evidence from Brazilian pork consumers. International Journal of Consumer Studies, v.35, p. 391-402, 2011.

BEN-SHAHAR, Omri; SCHNEIDER, Carl E. The failure of mandated disclosure. University of Michigan Law School, Empirical Legal Studies Cente. Working Paper, n. 10-008, 2010. Disponível em: <https://www.law. umich.edu/centersandprograms/lawandeconomics/abstracts/2010/Documents/10-008benshahar.pdf>. Acesso em: 9 jul. 2012.

BLACKMAN, Allen. Alternative pollution control policies in developing countries. Review of Environmental Economics and Policy, v. 4, n. 2, p. 234-253, 2010.

BLACKMAN, Allen; AFSAH, Shakeb; RATUNANDA, Damayanti. How do public disclosure pollution control programs work: evidence from Indonesia. Human Ecology Review, v.11, n. 3, p.235-246, 2004.

CAMPBELL, John L. Why would corporations behave in socially responsible ways? An institutional theory of corporate social responsibility. Academy of Management Review, v. 32, n. 3, p. 946-967, 2007.

CARRINGTON, Michal J.; NEVILLE, Benjamin A.; WHITWELL, Gregory J. Why ethical consumers don't walk their talk: Towards a framework for understanding the gap between the ethical purchase intentions and actual buying behaviour of ethically minded consumers. Journal of Business Ethics, v. 97, p. 139-158, 2010.

CIALDINI, Robert B.; RENO, Raymond R.; KALLGREN, Carl A. A focus theory of normative conduct: recycling the concept of norms to reduce littering in public places. Journal of Personality and Social Psychology, v. 58, n. 6, p. 1015-1026, 1990.

DASGUPTA, Susmita; LAPLANTE, Benoit; MAMINGI, Nlandu. Pollution and capital markets in developing countries. Journal of Environmental Economics and Management, v. 42, p. 310-335, 2001.

DASGUPTA, Susmita; WANG, Hua; WHEELER, David. Disclosure strategies for pollution control. In: TIETENBERG, Tom; FOLMER, Henk (Org.). The International Yearbook of Environmental and Resource Economics: a survey of current issues. Cheltenham: Edward Elgar, 2007. p. 93-119.

DOOREY, David J. Who made that? Influencing foreign labour practices through reflexive domestic disclosure regulation. Osgoode Hall Law Journal, v. 43, p. 353-405, 2005.

ESTLUND, Cynthia. Just the facts: the case for workplace transparency. Stanford Law Review, v. 63, p. 351408, 2011.

FERNANDES, Iêda; BRITO, Brenda; BARRETO, Paulo. Lições para divulgação da lista de infratores ambientais no Brasil. Revista de Direito Ambiental, v. 50, p. 81-101, abr.-jun. 2008.

GREENING; Daniel W.; TURBAN, Daniel B. Corporate social performance as a competitive advantage in attracting a quality workforce. Business e Society, v. 39, n. 3, p. 254-280, set. 2000. 
GUPTA; Shreekant; GOLDAR, Bishwanath. Do stock markets penalize environment-unfriendly behaviour? Evidence from India. Ecological Economics, v. 52, p. 81-95, 2005.

HALL, Peter A.; SOSKICE, David. An introduction to varieties of capitalism. In: HALL, Peter A.; SOSKICE, David (Org.). Varieties of Capitalism: the institutional foundations of comparative advantage. Nova York: Oxford University Press, 2001. p. 1-68.

HAMILTON, James T. Pollution as news: media and stock market reactions to the Toxic Releases Inventory data. Journal of Environmental Economics and Management, v. 28, p. 98-113, 1995.

HESS, David. The three pillars of corporate social reporting as new governance regulation: disclosure, dialogue and development. Michigan Ross School of Business, Working Paper, 2008. Disponível em: <http://deepblue.lib. umich.edu/bitstream/2027.42/60425/1/1112-DHess.pdf>. Acesso em: 10 ago. 2012.

KARKKAINEN, Bradley C. Information as environmental regulation: TRI and performance benchmarking, precursor to a new paradigm? Georgetown Law Journal, v. 89, p. 257-370, 2001.

KINDERMANN, Daniel. Why do some countries get CST sooner, and in greater quantity, than others? The political economy of corporate responsibility and the rise of market liberalism across the OECD: 19772007. WZB Discussion Paper. Disponível em: <http://ideas.repec.org/p/zbw/wzbkpw/spiii2009301.html>. Acesso em: 8 jun. 2012.

KONAR, Shameek; COHEN, Mark A. Information as regulation: the effect of community right to know laws on toxic emissions. Journal of Environmental Economics and Management, v.32, p. 109-124, 1997.

KONAR, Shammek; COHEN, Mark A. Does the market value environmental performance? The Review of Economics and Statistics, v. 83, n. 2, p. 281-289, 2001.

LANOIE, Paul; LAPLANTE, Benoît; ROY, Maité. Can capital markets create incentives for pollution control? Ecological Economics, v. 26, p. 31-41, 1998.

LANOU, Steven M. Production and organization learning: towards a new orientation for environmental policy. Massachusetts Institute of Technology, jun. 1998. Disponível em: <http://web.mit.edu/dusp/etpp/content/ projects/papers/Lanou\%20MIT\%20MCP\%20Thesis\%201998.pdf>. Acesso em: 17 jul. 2012.

LEE, E. Information disclosure and environmental regulation: green lights and gray areas. University of Hong Kong, 2010. Disponível em: <http://hub.hku.hk/bitstream/10722/125310/1/Content.pdf?accept=1>. Acesso em: 9 ago. 2012.

MACKEY, Alison; MACKEY, Tyson B.; BARNEY, Jay B. Corporate social responsibility and firm performance: investor preferences and corporate strategies. Academy of Management Review, v. 32, n. 3, p.817-835, 2007.

MARCHI, Scott; HAMILTON, James T. Assessing the accuracy of self-reported data: an evaluation of the toxics release inventory. Journal of Risk and Uncertainty, v. 32, p. 57-76, 2006.

NÖLKE, Andreas. A "BRIC" variety of capitalism and social inequality: the case of Brazil. Revista de Estudos e Pesquisas sobre as Américas, v. 4, n. 1, p. 1-14, 2010.

SAGE, William M. Regulating through information: disclosure laws and American health care. Columbia Law Review, v. 99, p. 1701-1829, 1999.

SCHATZ, Andrew. Regulating greenhouse gases by mandatory information disclosure. Virginia Environmental Law Journal, v. 26, p. 335-393, 2008.

SCHNEIDER, Ben Ross. Comparing capitalisms: liberal, coordinated, network, and hierarchical varieties. 2008. Disponível em: <http://www.ces.fas.harvard.edu/events/papers/Schneider_Comparing_Capitalisms.pdf>. Acesso em: 20 jun. 2012. 
SCHNEIDER, Ben Ross. Hierarchical market economies and varieties of capitalism in Latin America. Journal of Latin American Studies, v. 41, n. 3, p. 553-575, 2009.

SHANE, Philip B.; SPICER, Barry H. Market response to environmental information produced outside the firm. The Accounting Review, v. 98, n. 3, p, 521-538, jul. 1983.

SIMON, Herbert A. A behavioral model of rational choice. Quarterly Journal of Economics, v. 69, p. 99-118, fev. 1955.

STEPHAN, Mark. Environmental information disclosure programs: they work, but why? Social Science Quarterly, v. 83, n. 1, mar. 2002.

SUCHMAN, Mark C. Managing legitimacy: strategic and institutional approaches. Academy of Management Review, v. 20, n. 3, p. 571-610, 1995.

TIETENBERG, Thomas H. Emissions trading: an exercise in reforming pollution policy. Washington, DC: Resources for the Future, 1985.

TIETENBERG, Tom. Disclosure strategies for pollution control. Environmental and Resource Economics, v. 11, n. 3-4, p. 587-602, 1998.

VAN ERP, Judith. Reputational sanction in private and public regulation. Erasmus Law Review, v. 1, n. 5, p. 145-162, 2008.

WANG, Hua; BI, Jun; WHEELER, David et al. Environmental performance rating and disclosure: China's GreenWatch Program. Journal of Environmental Management, v. 71, p. 123-133, 2004.

WEIL, David; FUNG, Archon; GRAHAM, Mary et al. The effectiveness of regulatory disclosure policies. Journal of Policy Analysis and Management, v. 25, n. 1, p.155-181, 2006.

ZANITELLI, Leandro Martins. Capitalismo brasileiro e responsabilidade social empresarial. Sequência, v. 34, n. 66, p. 83-112, 2013. 
Para publicar na revista Brasileira de Políticas Públicas, acesse o endereço eletrônico www.rbpp.uniceub.br

Observe as normas de publicação, para facilitar e agilizar o trabalho de edição. 